\title{
Microscopic origin of the Bekenstein-Hawking entropy
}

\section{Citation}

Strominger, Andrew, and Cumrun Vafa. 1996. "Microscopic Origin of the Bekenstein-Hawking Entropy." Physics Letters B 379 (1-4): 99-104. https://doi.org/10.1016/0370-2693(96)00345-0.

\section{Permanent link}

http://nrs.harvard.edu/urn-3:HUL.InstRepos:41385003

\section{Terms of Use}

This article was downloaded from Harvard University's DASH repository, and is made available under the terms and conditions applicable to Other Posted Material, as set forth at http:// nrs.harvard.edu/urn-3:HUL.InstRepos:dash.current.terms-of-use\#LAA

\section{Share Your Story}

The Harvard community has made this article openly available.

Please share how this access benefits you. Submit a story.

\section{Accessibility}


HUTP-96/A002

RU-96-01

hep-th/9601029

\title{
Microscopic Origin of the Bekenstein-Hawking Entropy
}

\author{
Andrew Strominger \\ Department of Physics \\ University of California \\ Santa Barbara, CA 93106-9530 \\ and \\ Cumrun Vafa \\ Lyman Laboratory of Physics \\ Harvard University \\ Cambridge, MA 02138
}

\begin{abstract}
The Bekenstein-Hawking area-entropy relation $S_{B H}=A / 4$ is derived for a class of five-dimensional extremal black holes in string theory by counting the degeneracy of BPS soliton bound states.
\end{abstract}




\section{Introduction}

In the early seventies a sharp and beautiful analogy was discovered between the laws of black hole dynamics and the laws of thermodynamics [1 17 . In particular the BekensteinHawking entropy - one quarter the area of the event horizon - behaves in every way like a thermodynamic entropy. A missing link in this circle of ideas is a precise statistical mechanical interpretation of black hole entropy. One would like to derive the BekensteinHawking entropy - including the numerical factor - by counting black hole microstates. The laws of black hole dynamics could then be identified with - and not just be analogous to - the laws of thermodynamics.

In this paper progress in this direction is reported. We consider phases of string theory with five noncompact dimensions and $N=4$ supersymmetryl, (e.g. type II string theory on $K 3 \times S^{1}$ or heterotic string theory on $T^{5}$ ). Black holes in these theories can carry both an axion charge $Q_{H}$ and an electric charge $Q_{F}$ 2. Extremal black holes with either $Q_{H}=0$ (fundamental heterotic string states) or $Q_{F}=0$ (but not both) have degenerate horizons with zero area. We accordingly look for BPS saturated states - i.e. extremal black holes - for which both $Q_{F}$ and $Q_{H}$ are non-vanishing. Such BPS states preserve only $1 / 4$ of the $N=4$ supersymmetry. They may be viewed as bound states of minimallycharged BPS solitons, and their exact degeneracy as a function of $Q_{F}$ and $Q_{H}$ can be topologically computed by counting soliton bound states. In particular we show that the leading degeneracy for the logarithm of the bound-state degeneracy for large $Q_{H}$ and fixed $Q_{F}$ is given by 3

$$
S_{\text {stat }}=2 \pi \sqrt{Q_{H}\left(\frac{1}{2} Q_{F}^{2}+1\right)}
$$

On the other hand we will find that the Bekenstein-Hawking entropy as determined from the low-energy effective action is

$$
S_{B H}=2 \pi \sqrt{\frac{Q_{H} Q_{F}^{2}}{2}}
$$

in agreement with (1.1) for large charges.

1 Analogous results follow for $N=8$ as indicated below.

${ }^{2} Q_{F} \in \Gamma^{5,21}$ where $\Gamma^{5,21}$ is the Narain lattice of heterotic strings compactified down to 5 dimensions and $Q_{F}^{2}=Q_{R}^{2}-Q_{L}^{2}$.

3 Given the $O(21,5)$ invariance of the theory one expects that the bound-state degeneracy of these BPS solitons be a functions of $Q_{F}^{2}$ and $Q_{H}$. 
The five-dimensional problem is considered here because it seems to be the simplest non-trivial case. We expect that similar calculations will reproduce $S_{B H}$ for other types of black holes in string theory. Previous attempts at a microscopic derivation of $S_{B H}$ include [8-18].

In section 2 we present the black hole solutions and compute the area of their event horizons. In section 3 the bound state degeneracy is asymptotically computed by relating it to the elliptic genus of a certain two-dimensional sigma model. We conclude with discussion in section 4 .

\section{A Class of Five Dimensional Extremal Black Holes}

The low energy action for type II string theory compactified on $K 3 \times S^{1}$ contains the terms

$$
\frac{1}{16 \pi} \int d^{5} x \sqrt{-\tilde{g}}\left(e^{-2 \phi}\left(R+4(\nabla \phi)^{2}-\frac{1}{4} \tilde{H}^{2}\right)-\frac{1}{4} F^{2}\right)
$$

in the string frame. We adopt conventions in which $\alpha^{\prime}=G_{N}=1 . F$ is a RR 2-form field strength (associated with the right-moving current algebra in the dual heterotic picture) and $\tilde{H}$ is a 2-form axion field strength arising from the NS-NS 3-form with one compnent tangent to the $S^{1}$. We work on a submanifold of the Narain moduli space for $K 3 \times S^{1}$ on

which nonzero $F$ does not require nonconstant moduli. In the Einstein frame $\left(g=e^{-4 \phi / 3} \tilde{g}\right)$ (2.1) becomes

$$
\frac{1}{16 \pi} \int d^{5} x \sqrt{-g}\left(R-\frac{4}{3}(\nabla \phi)^{2}-\frac{e^{-4 \phi / 3}}{4} \tilde{H}^{2}-\frac{e^{2 \phi / 3}}{4} F^{2}\right) .
$$

A black hole can carry electric charge with respect to both $F$ and $\tilde{H}$,

$$
\begin{aligned}
& Q_{H} \equiv 1 / 4 \pi^{2} \int_{S^{3}} * e^{-4 \phi / 3} \tilde{H}, \\
& Q_{F} \equiv 1 / 16 \pi \int_{S^{3}} * e^{2 \phi / 3} F .
\end{aligned}
$$

For the spherically symmetric configurations that we consider this implies

$$
\begin{aligned}
* e^{-4 \phi / 3} \tilde{H} & =2 Q_{H} \epsilon_{3}, \\
* e^{2 \phi / 3} F & =\frac{8 Q_{F}}{\pi} \epsilon_{3},
\end{aligned}
$$


where $\epsilon_{3}$ is the volume element on the unit $S^{3}$. We have chosen our conventions so that $Q_{H}$ and $\frac{1}{2} Q_{F}^{2}$ are integers 1 . $O(21,5)$ invariance of the full lagrangian (which includes 26 gauge fields) implies that all of the following formulae remain valid with the replacement $Q_{F}^{2}=Q_{R}^{2}-Q_{L}^{2}$.

An extremal black hole carrying both types of charges can have an event horizon with nonzero area. The near-horizon geometry will be the five-dimensional $A d S_{2} \times S^{3}$ charged Robinson-Berttoti universe with constant dilaton $\phi=\phi_{h}$. The constant value $\phi_{h}$ is determined in terms of the charges by the dilaton equation of motion

$$
16 \nabla^{2} \phi+2 e^{-4 \phi / 3} \tilde{H}^{2}-e^{2 \phi / 3} F^{2}=0 .
$$

Substituting $\phi=\phi_{h}$ and (2.4) this implies

$$
e^{2 \phi_{h}}=\frac{1}{2}\left(\frac{4 Q_{F}}{\pi Q_{H}}\right)^{2}
$$

Note that the type II closed string coupling at the horizon is weak when the ratio $Q_{F} / Q_{H}$ is small. If the asymptotic value of $\phi_{\infty}$ of the dilaton is tuned to coincide with the special value determined by (2.6) then the dilaton is everywhere constant and Einstein's equation becomes

$$
\begin{aligned}
R_{a b} & =3\left(\frac{8 Q_{H} Q_{F}^{2}}{\pi^{2}}\right)^{2 / 3}\left(\epsilon_{3 a c d} \epsilon_{3 b}{ }^{c d}-g_{a b}\right) \\
\phi & =\phi_{h} .
\end{aligned}
$$

This is just the equation for $d=5$ Reissner-Nordstrom with charge $\sqrt{3}\left(\frac{8 Q_{H} Q_{F}^{2}}{\pi^{2}}\right)^{1 / 3}$. The extremal solution can be found for example in [19]:

$$
d s^{2}=-\left(1-\left(\frac{r_{0}}{r}\right)^{2}\right)^{2} d t^{2}+\left(1-\left(\frac{r_{0}}{r}\right)^{2}\right)^{-2} d r^{2}+r^{2} d \Omega_{3}^{2},
$$

where

$$
r_{0}=\left(\frac{8 Q_{H} Q_{F}^{2}}{\pi^{2}}\right)^{1 / 6} .
$$

$S^{1}$ reduction of this solution to $d=4$ gives the dyonic solution discussed in reference [20], where it is further shown that the resulting configurations are annihilated by one quarter of the supersymmetries (This is also evident from the stringy description given below).

\footnotetext{
$4 Q_{F}^{2}=2$ for a minimally-charged perturbative heterotic string state, while $Q_{H}=1$ for a minimally-charged heterotic fivebrane which wraps $T^{5}$ once.
} 
The Einstein-frame area of the extremal black hole horizon is given by the volume of the $S^{3}$ :

$$
\text { Area }=8 \pi \sqrt{\frac{Q_{H} Q_{F}^{2}}{2}} .
$$

The Bekenstein-Hawking entropy is

$$
S_{B H}=2 \pi \sqrt{\frac{Q_{H} Q_{F}^{2}}{2}} .
$$

Even if the asymptotic value of the dilaton $\phi_{\infty} \neq \phi_{h}$, the near-horizon RobinsonBerttoti geometry is still constrained to obey (2.6) and (2.7). Hence as the asymptotic value of the fields are adiabatically changed, the near horizon geometry is unaltered. This type of behavior has been noticed previously in families of exact solutions with generic asymptotic moduli (see for example [21,17, 22, 18]) and can be intuitively understood [23] by viewing black holes as solitons which interpolate between maximally symmetric vacua at infinity and the horizon (in our case the $d=5$ Robinson-Berttoti vacuum). In conclusion the dilaton-independent relation (2.11) is valid even when $\phi_{\infty} \neq \phi_{h}$.

The action (2.1) as well as the entropy (2.11) receives corrections from both string loop and sigma model perturbation theory. $N=4$ nonrenormalization theorems ensure that there are no corrections to the lowest dimension terms exhibited in (2.1), but higher dimension terms will be corrected in general. Type II string loop corrections are suppressed by powers of $g_{I I} \sim Q_{F} / Q_{H}$. Sigma model corrections are suppressed by inverse powers of the string-frame Schwarzchild radius, which is $\tilde{r}_{0 I I} \sim \sqrt{Q_{F}^{2} / Q_{H}}$. in the type II theory requires that both $Q_{H}$ and $Q_{F}$ are large. String dualities of various kinds might be used to extend the range of validity of (2.11).

\section{Counting of Microscopic BPS States}

The counting of microscopic BPS states has become possible for type II string compactifications thanks to recent progress in understanding non-perturbative string theory. Of particular importance is the beautiful identification of D-branes [24,25] as the source of BPS states carrying $Q_{F}$ Ramond-Ramond charge [26] and the relation between counting bound states of D-branes and specific questions in certain quantum field theories on the D-brane worldvolume [27,28, 29, 30,31].

${ }^{5}$ For the dual heterotic theory, $g_{h}^{2} \sim\left(Q_{H} / Q_{F}\right)$ and $\tilde{r}_{0 h} \sim \sqrt{Q}_{H}$. 
Consider type IIB string theory compactified on $K 3 \times S^{1}$. Type IIB string theory has $p$-brane solitonic states for odd values of $p$ [32]. We consider D-branes with $p=1,3,5$ wrapped around $S^{1} \times C$ where $C$ is a supersymmetric (i.e. holomorphic) 0-, 2- or 4-cycle of $K 3$. These states carry the Ramond-Ramond charge $Q_{F}$, and $Q_{F}^{2}$ is the self-intersection number of the collection of cycles [28,30,29,31]. It was argued in [27] that BPS states in spacetime which preserve half of the spacetime supersymmetries correspond to supersymmetric ground states of the D-brane worldvolume theory. This follows directly from the fact that worldvolume supersymmetries arise as the projection of unbroken spacetime supersymmetries. This observation was generalized in [31] to spacetime BPS states, which preserve fewer spacetime supersymmetries. The corresponding states of the D-brane worldvolume theory correspond to worlvolume BPS states, which preserve fewer worldvolume supersymmetries than the worldvolume ground states. Since we are interested in states which preserve $1 / 4$ of the spacetime supersymmetries we should count BPS states which preserve $1 / 2$ of the supersymmetries of the relevant D-brane worldvolume theory. Let us first consider a limit in which the worldvolume theory of the D-brane simplifies. Consider the limit in which the $K 3$ is small compared to the size of the circle $S^{1}$. In this limit we get an effectively two dimensional worldvolume theory on $S^{1} \times R$ (where $R$ corresponds to time). Based on the expected ground state degeneracies of the corresponding effective theory 6 it was conjectured in [29] that this theory is a supersymmetric sigma model whose target space is the symmetric product of $\frac{1}{2} Q_{F}^{2}+1$ copies of $K 3$

$$
M=\frac{(K 3)^{\otimes\left[\frac{1}{2} Q_{F}^{2}+1\right]}}{S_{\left[\frac{1}{2} Q_{F}^{2}+1\right]}}
$$

where $S_{n}$ is the permutation group on $n$ objects. Subsequently this was verified [30] at least for cases where $Q_{F}$ comes from primitive 2-cycles in $K 3$ or from $\left[\frac{1}{2} Q_{F}^{2}+1\right] 0$-cycles together with one 4-cycle in $K 3$ (for 2-cycles this was rephrased and further checked as a counting problem for rational curves on $K 3$ with double points [33]). In the latter case the origin of (3.1) can be simply understood as the moduli space of $\frac{1}{2} Q_{F}^{2}+1$ unordered points on $K 3$. The conjecture (3.1) was further verified in [31] in cases where there are more than one 4-cycle and some 0-cycles (and was connected to the strong coupling test of

6 According to string duality this is expected to be the same as the oscillator degeneracy of bosonic strings. 
Olive-Montonen duality on $K 3$ [34]). For the purposes of this paper consideration of any of these configurations of D-branes sufficest.

Thus we have to count the BPS states of a supersymmetric sigma model on $M$ if we wish to count states which preserve only $1 / 4$ of the spacetime supersymmetries. But these are precisely those states which are killed by (say) the right-moving supercharge, with no restrictions on the left-movers. In other words we consider RR sector states of this sigma model in their right-moving vacuum, i.e., $\bar{L}_{0}=\frac{1}{2}(H-P)=0$ and arbitrary $L_{0}=\frac{1}{2}(H+P)$. The generating function for the degeneracies of such states is bounded by the elliptic genus of the sigma model on $M$ [35,36] which is computable for all manifolds $M$. The actual number of BPS states may depend on the moduli of $K 3$, but the elliptic genus, which is the appropriate weighted sum (with \pm 1 ), is moduli independent. It is tempting to speculate that the elliptic genus is the more relevant quantity which appears in physical quantities (just as was the case considered in [37]) but either quantity will give the same leading degeneracy as a function of charges [38], and this distinction is unimportant for our purposes in this paper. Note that the eigenvalues of $L_{0}$ contributing to the elliptic genus are restricted to be integers because $L_{0}=L_{0}-\bar{L}_{0}=P$ where $P$ is the momentum operator on the $S^{1}$.

Before considering the degeneracy of these states let us see what charges they carry. In addition to $Q_{F}$ charge they carry a charge corresponding to momentum $P$ around $S^{1}$. If we go from type IIB to type IIA by dualizing the $S^{1}$, these states carry $P$ units of winding around $S^{1}$, i.e. they have $P$ units of electric charge with respect to $B_{\mu \theta}$ where $\theta$ corresponds to the circle direction and $\mu$ denotes the five dimensional spacetime indices, i.e. $Q_{H}=P$. Thus the BPS states of the D-brane worldvolume theory we are considering carry precisely the charges $Q_{F}$ and $Q_{H}$ for which the corresponding extremal black hole solutions were found in the previous section.

To compare their degeneracy with the Bekenstein-Hawking entropy (2.11), which is expected to be accurate for large $Q_{H}$ and $Q_{F}^{2}$, all we need to do is to consider the asymptotic degeneracy of the above BPS states for large $Q_{H}=P$ and $Q_{F}^{2}$ (even though it is straight forward to compute it for all $Q_{H}$ and $Q_{F}^{2}$ by an orbifold computation). For a hyperkahler manifold $M$ of dimension $4 k$ we have a sigma model with central charge $c=6 k$. The

7 In fact all we will need is that the dimensions of $M$ grows as $4\left(\frac{1}{2} Q_{F}^{2}+1\right)$, which is much easier to argue [30,31]. 
left-moving oscillator degeneracy for unitary conformal theories at level $L_{0}=n$ goes for $n>>1$ as 39

$$
d(n, c) \sim \exp \left(2 \pi \sqrt{\frac{n c}{6}}\right)
$$

In our case

$$
\begin{aligned}
c & =6\left(\frac{1}{2} Q_{F}^{2}+1\right), \\
n & =Q_{H},
\end{aligned}
$$

so we get for the growth of the elliptic genus, or equivalently the degeneracy of BPS solitons for $Q_{H}>>1$

$$
S_{\text {stat }}=\ln d\left(Q_{F}, Q_{H}\right) \sim 2 \pi \sqrt{Q_{H}\left(\frac{1}{2} Q_{F}^{2}+1\right)} .
$$

This agrees to leading order with the expected Bekenstein-Hawking entropy (2.11) for large $Q_{F}^{2}$, in which case $(2.11)$ is reliable

\section{Discussion}

In the presence of $N$ D-branes, the open string sector of perturbation theory involves an expansion in $g_{I I} N$ (where $g_{I I}$ is the asymptotic value of the type II closed string coupling) because holes in the string world sheet can have $N$ types of Dirichlet boundary conditions. The effective value of $N$ for our configurations grows like $\frac{1}{2} Q_{F}^{2}$. Hence for small but fixed $g_{I I}$ string perturbation theory will break down for sufficiently large charge. The correct physical picture of the objects we discuss really is as a large semiclassical black hole with an event horizon. The description as a supersymmetric cycle embedded in $K 3$ suffers large quantum corrections. It nevertheless can be reliably used to compute the asymptotic degeneracy of BPS states because that is a topological quantity related to the elliptic genus.

The validity of string perturbation theory can be restored by taking $g_{I I}$ to be very small - smaller than $1 / N$. In this case string perturbation theory is valid, and the physical

8 It would be interesting to understand large $c$ corrections to this formula in order to determine the range of validity of our estimate.

9 This result can also be derived for an $N=8$ toroidal type II compactification, in which case the same type of D-brane configuration breaks $7 / 8$ of the supersymmetry. The only difference in the derivation is that $K 3$ is replaced by $T 4$ in the symmetric product in (3.1). Since the dimension of the resulting $M$ is the same this does not affect the growth of the elliptic genus. We thank A. Sen for discussions on this point. 
picture of the BPS state as a supersymmetric $K 3$ cycle is the correct weakly-coupled description. For such very small $g_{I I}$, the string length becomes larger than the Schwarzchild radius (equation (2.9)). Hence the black hole picture will suffer large stringy corrections.

So we have a BPS state which at very weak coupling is described by $p$-branes wrapping supersymmetric $K 3$ cycles, but at strong coupling transforms into a hole in spacetime!

We believe that our results will have implications for the black hole information puzzle. A central theme in studies of this puzzle over the last several years has been the problem of low-energy scattering of ordinary quanta by an extremal black hole [40, 41, 42, 43, 44, Naively this process proceeds by absorption followed by Hawking reemission, and so the question of unitarity violation arises.

In principle light might be shed on this puzzle by employing D-brane technology [26, 45] to compute the scattering. However one immediately encounters the above-mentioned problem that string theory is strongly coupled in the region of interest. Perhaps a string duality can be used to map it to a weakly-coupled problem. In any case it is hard to imagine how any calculation based on our D-brane description of the extremal black hole could yield a non-unitary answer. However the alternatives are highly constrained by lowenergy consistency. Two of the alternatives involve low-energy effective non-locality, as advocated for example in [4], or a very long scattering time, as advocated for example in [47]. It is also very hard to imagine how either of these features could emerge in a D-brane description. Our results of so far are consistent with all of these proposals, and do not tell us definitively how string theory solves the information puzzle. Nevertheless we have more clues and are optimistic that further progress on this issue is now possible.

We could consider other compactifications-for example heterotic string compactified on $T^{6}(N=4)$ or on $K 3 \times T^{2}(N=2)$, which are dual to type II strings on $K 3 \times T^{2}$ or on Calabi-Yau. The Bekenstein-Hawking entropy for the $N=4$ cases have been computed in [19,20,18], while $N=2$ cases appear in [21]. It is not too difficult in these examples to set up the computation for the BPS states which preserve only one unit of supersymmetry. In one formulation, it is related to the study of cohomology of moduli space of stable holomorphic $S U(N)$ bundles on the six-manifold with fixed second and third Chern classes determined by the charges. Unfortunately at the present the dimension of the cohomology of such moduli spaces is not known. It would be interesting to compute the growth of the cohomology of the moduli spaces for these bundles on the six-manifolds and check the prediction obtained from the Bekenstein-Hawking entropy.

\section{Acknowledgements}


We would like to thank G. Horowitz, J. Polchinski, S. Shenker and E. Witten for valuable discussions. C.V. also thanks the hospitality of Rutgers University where this work was completed. The research of C.V. is supported in part by NSF grant PHY-9218167. The research of A.S. is supported in part by DOE grant DOE-91ER40618. 


\section{References}

[1] D. Christodolou, Phys. Rev. Lett. 25, (1970) 1596; D. Christodolou and R. Ruffini, Phys. Rev. D4, (1971) 3552.

[2] R. Penrose and R. Floyd, Nature 229 (1971) 77.

[3] S. Hawking, Phys. Rev. Lett. 26, (1971) 1344.

[4] J. Bekenstein, Lett. Nuov. Cimento 4 (1972) 737, Phys. Rev. D7 (1973) 2333, Phys. Rev. D9 (1974) 3292.

[5] B. Carter, Nature 238 (1972) 71.

[6] J. Bardeen, B. Carter and S. Hawking, Comm. Math. Phys. 31 (1973) 161.

[7] S. Hawking, Nature 248 (1974) 30, Comm. Math. Phys. 431975.

[8] J. Bekenstein, Phys. Rev D12 (1975) 3077.

[9] S. Hawking, Phys. Rev D13 (1976) 191.

[10] W. Zurek and K. Thorne, Phys. Rev. Lett. 54, (1985) 2171.

[11] G. 'tHooft, Nucl. Phys. B335 (1990) 138; Phys. Scr. T36 (1991) 247.

[12] L. Susskind, hep-th/9309145.

[13] L. Susskind and J. Uglum, hep-th/9401070, Phys. Rev. D50 (1994) 2700.

[14] C. Teitelboim, hep-th/9510180.

[15] A. Sen, hep-th/9504147.

[16] S. Carlip, gr-qc/9509024.

[17] F. Larsen and F. Wilczek, hep-th/9511064.

[18] M. Cvetic and A. Tseytlin, hep-th/9512031.

[19] G. Gibbons, Nucl. Phys. B207 (1982) 337; G. Gibbons and K. Maeda Nucl. Phys. B298 (1988) 741.

[20] R. Kallosh, A. Linde, T. Ortin, A. Peet and A. van Proeyen, Phys. Rev. D46 (1992) 5278.

[21] S. Ferrara, R. Kallosh and A. Strominger, hep-th/9508072, Phys. Rev. D 52, (1995) 5412 .

[22] M. Cvetic and D. Youm, hep-th/9507090.

[23] G. Gibbons and P. Townsend, Phys. Rev. Lett. 71 (1993) 3754.

[24] J. Dai, R. Leigh and J. Polchinski, Mod. Phys. Lett. A4 (1989) 2073.

[25] P. Horava, Phys. Lett. B231 (1989) 251.

[26] J. Polchinski, hep-th/9510017.

[27] E. Witten, hep-th/9510135.

[28] A. Sen, hep-th/9510229, hep-th/9511026

[29] C. Vafa, hep-th/9511088.

[30] M. Bershadsky, V. Sadov and C. Vafa, hep-th/9511222.

[31] C. Vafa, hep-th/9512078.

[32] G. Horowitz and A. Strominger, Nucl. Phys. B360 (1991) 197. 
[33] S.-T. Yau and E. Zaslow, hep-th/9512121.

[34] C. Vafa and E. Witten, Nucl. Phys. B431 (1994) 3.

[35] E. Witten, Comm. Math. Phys. 109 (1987) 525.

[36] A.N. Schellekens and N.P. Warner, Phys. Lett. B177 (1986) 317.

[37] J. Harvey and G. Moore, hep-th/9510182.

[38] I. Kani and C.Vafa, Comm. Math. Phys. 130 (1990) 529.

[39] J.L. Cardy, Nucl. Phys. B270 (1986) 186.

[40] J. Preskill, P. Schwarz, A. Shapere, S. Trivedi and F. Wilczek, Mod. Phys. Lett. A6 (1991) 2353.

[41] C. Callan, S. Giddings, J. Harvey, and A. Strominger, Phys. Rev. D45 (1992) R1005.

[42] A. Strominger and S. Trivedi, Phys. Rev. D48 (1993) 5778.

[43] S. Giddings, Phys. Rev D49 (1994) 4078.

[44] P. Kraus and F. Wilczek, hep-th/9411219, Nucl. Phys. B433 (1995) 403.

[45] C. Callan and I. Klebanov, hep-th/9511173.

[46] L. Susskind, Phys. Rev. Lett. 71, (1993) 2367; L. Susskind and L. Thorlacius, Phys. Rev. D49 (1994) 966; L. Susskind, ibid. 6606.

[47] J. Polchinski and A. Strominger, hep-th/9407008, Phys. Rev. D50 (1994) 7403. 